\title{
MODOS RESONANTES DE LA BAHIA DE TODOS SANTOS EXCITADOS POR EL TSUNAMI DEL 22 DE MAYO DE 1960.
}

\section{por}

Noemi Yolanda Rivera Fernández

Escuela Superior de Ciencias Marinas Universidad Autónoma de Baja California Apartado Postal 453

Ensenada, Baja California, México

$$
\text { y }
$$

Hugo Gustavo Peña

Centro de Investigación Cientifica y de Educación Superior de Ensenada, B.C. Espinoza No. 843

Ensenada, Baja California, México

\section{RESUMEN}

En este trabajo se obtienen los modos de oscilaciôn de la Bahía de Todos Santos, Ensenada, B.C., excitados por el tsunami del 22 de mayo de 1960. Se efectúa el análisis espectral de la serie de datos obtenida del registro mareográfico. Se aplica el Método de Máxima Entropia determinándose los siguientes períodos de resonancla: $45.53 \mathrm{~min}, 26.79 \mathrm{~min}$ y 13.11 min.

Con el objeto de apoyar estos resultados, se utiliza un modelo teórico el cual toma en cuenta una bahía de forma rectangular, con anchura constante y variación lineal de 1 a profundidad con una profundidad promedio en 1a boca. Se incluye también, un factor de fricción.

La comparación entre los modos de oscilación observados y calculados resulta ser adecuada, concluyêndose que el modelo predice razonablemente bien el comportamiento dinámico de la bahfa en sus características resonantes.

\section{ABSTRACT}

In this paper, the normal oscillation modes of the Todos Santos Bay, Ensenada, B.C. excited by the Chilean tsunami of May 22, 1960 are obtained. A tide gauge record is analized and processing by Maximum Entropy Espectral Method. The resultant resonant periods are $45.53 \mathrm{~min}, 26.79 \mathrm{~min} y$ $13.11 \mathrm{~min}$. 
A simple mathematical model for free oscillations on a bay of rectangular shape and linear variation of depth are used. A frictional factor is also included.

The agreement of the mathematical model with the normal modes observed is very good. This demostrates that the tide gauge record analysis responds for the dimensional characteristics of the bay.

\section{INTRODUCCION}

Los puertos y bahías con comunicaciôn abierta al océano, se encuentran expuestos a perturbaciones producidas por ondas incidentes que pueden provocar amplificaciones en el nivel medio del mar ocasionando graves daños a las embarcaciones ancladas, a las estructuras portuarias y a las poblaciones costeras.

Las ondas que 1legan a una cuenca pueden inducirla a oscilar constituyendo un sistema dinámico oscilante. Si el período de uno de los modos de oscilación de la cuenca coincide o está cercano al período de las ondas incidentes, se producirá el fenómeno de resonancia que causará un aumento de la amplitud de oscilación dentro de la cuenca.

Todos los cuerpos de agua, ya sea el océano, plataforma continental, bahías, puertos, etc., tienen sus modos de oscilación característicos que dependen de su geometría. Una manera de determinar estos modos de oscilación es a través de 1a respuesta del cuerpo de agua a perturbaciones provocadas por algún fenómeno, como las fluctuaciones de la presión atmosférica, ondas de tsunami, etc. Una onda de tsunami es originada por deslizamientos de tierra o por sismos, y aunque son de carácter impulsivo, sus efectos sobre las costas pueden durar del orden de una semana.

En este trabajo se considera una onda de tsunami como el mecanismo causante de la perturbación, que es notada en el registro mareográfico en el cual se observa la presencia de oscilaciones resonantes.

Se han elaborado numerosos trabćjos teóricos y experimentales acerca de oscilaciones inducidas en cuencas de diferentes geometrías, simples, complejas y con comunicación al océano. Este problema también se ha tratado en cuencas de geometria irregular, aunque desarrollar un procedimiento de cálculos prácticos es difícil por el hecho de que las ondas incidentes no son periódicas, y por la configuración de la cuenca. Aquí se considera que la bahỉa es de forma rectangular y que su anchura es constante, por lo que el problema se simplifica.

Para la zona de Ensenada, el primer trabajo de este tipo fué el de Raichlen (1970), quién determinó espectros de potencia para los tsunamis de mayo de 1960 y marzo de 1964. 
Posteriormente, Sánchez (1980) efectúa un estudio de tsunamis para los puertos de la costa occidental de México, obtiene los modelos de oscilactón para la plataforma adyacente al puerto y hace un análisis espectral de varios tsunamis.

DESCRIPCION DE LA ZONA DE ESTUDIO

La Bahía de Todos Santos se localiza sobre la costa oeste de la Peninsula de Baja California (Fig. 1) entre los $31^{\circ} 40^{\prime}$ y $31^{\circ} 55^{\prime} \mathrm{N}$ y entre los $116^{\circ} 36^{\prime}$ y $116^{\circ} 50^{\prime} \mathrm{O}$. La topografía de la bahía se muestra en la figura 2, notándose que las isobatas superiores a $10550 \mathrm{~m}$ son irregulares y que sólo a partir de las isobatas de $15 \mathrm{~m}$ tienden a ser paralelas a la lînea de costa, especialmente en el märgen Este que estâ constituído por playas arenosas.

\section{DATOS UTILIZADOS}

Para la aplicación del modelo se consideran ondas largas sinusoidales penetrando del mar hacia la bahía perpendicularmente a la lînea de costa; movimiento del agua armónico y. función de una coordenada longitudinal y del tiempo, variaciones graduales en sección transversal, presión hidrostática y linealidad en la ecuación dinámica con un efecto de fricción lineal (Dorrestein, 1961).

Las ecuaciones que gobiernan el movimiento del nivel del mar dentro de la bahỉa son: la ecuación de continuidad:

$$
\frac{\partial q}{\partial x}+\frac{b \partial h}{\partial x}=0
$$

y la ecuación dinâmica:

$$
\frac{\partial v}{\partial t}=-g \frac{\partial h}{\partial x}-f w v
$$

donde

$$
\begin{aligned}
\mathrm{b}(\mathrm{x})= & \text { anchura de la sección transversal } \\
\mathrm{d}(\mathrm{x})= & \text { profundidad promedio de la sección transversal } \\
f= & \text { parâmetro de fricción adimensional } \\
\mathrm{g}= & \text { aceleraciốn de la gravedad } \\
\mathrm{h}(\mathrm{x}, \mathrm{t})= & \text { altura de la superficie del agua sobre la posi- } \\
& \text { ción cero. } \\
\mathrm{q}(\mathrm{x}, \mathrm{t})= & \text { descarga a través de una sección transversal } \\
\mathrm{v}(\mathrm{x}, \mathrm{t})= & \text { q/bd= media seccional de la velocidad longitu- } \\
& \text { dinal del fluído } \\
\mathrm{x}= & \text { coordenada longitudinal positiva hacia el mar } \\
\omega= & \text { frecuencia angular del movimiento de agua }
\end{aligned}
$$

Haciendo una transformación de las ecuaciones (1) y (2) y un cambio de la variable $x$ por $s$, y de la variable $h$ por $H$, se obtiene la siguiente ecuación: 

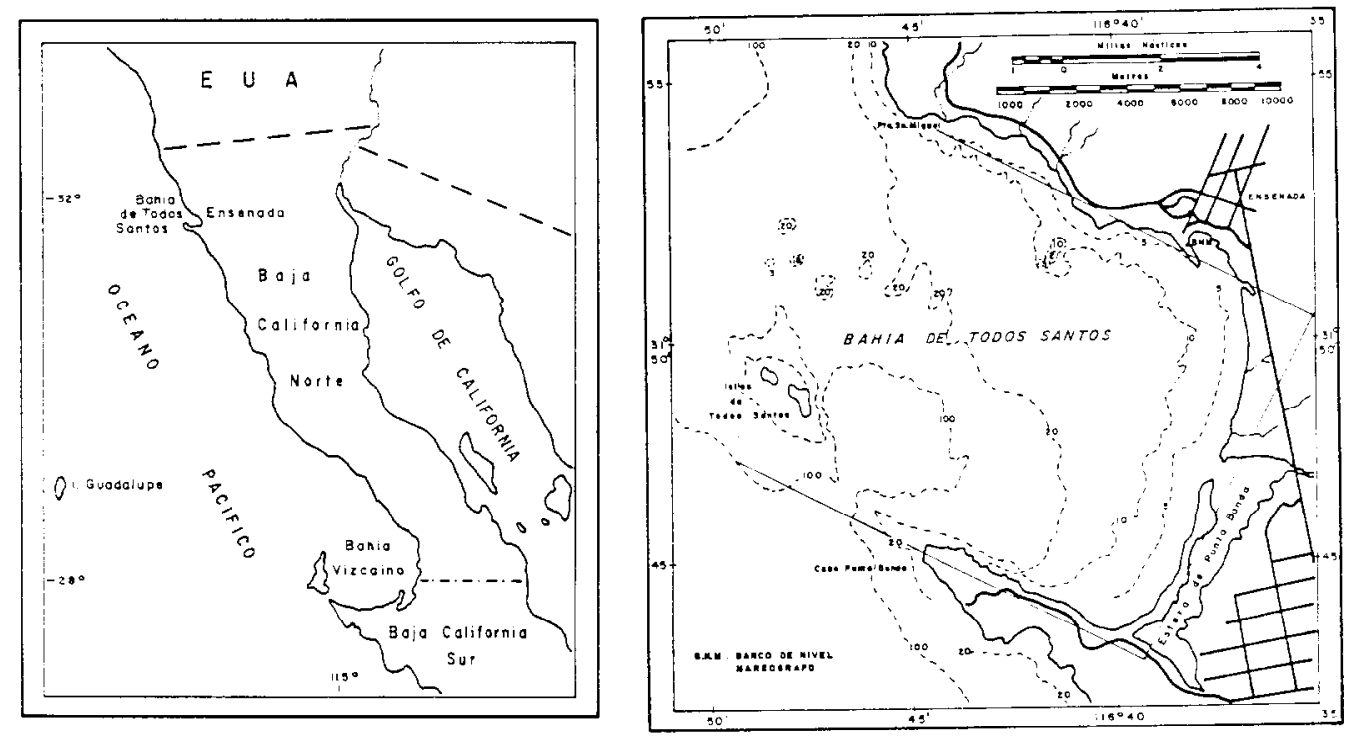

Fig. 2. BATIMETRIA Y ESQUEMATIZACION DE LA BAHIA

Fig.- 1. LOCALIZACION DE LA ZONA DE ESTUDIO (BAHIA

DE TODOS SANTOS (PROFUNDIDAD EN BRAZAS). JE TODOS SANTOS $31^{\circ} 40^{\prime}$ A $31^{\circ} 50^{\prime}$ Y $116^{\circ} 36^{\prime}$ $A 116^{\circ} 50^{\prime} W$ ) 


$$
\frac{d^{2} H}{d s^{2}}+\frac{1}{b \sqrt{d}} \frac{d}{d s}(b \sqrt{d}) \cdot \frac{d H}{d s}+{ }^{H}=0
$$

la cual es una ecuación diferencial de segundo orden por 1o que su solución estará dada por una superposición lineal de dos funciones de $s$ mutuamente independientes.

$$
\begin{aligned}
& \text { La nueva variable } s \text { estará dada por: } \\
& \mathrm{s}=\int_{0}^{\mathrm{x}} \mathrm{dx} \sqrt{\frac{1-\text { if }}{\mathrm{gd}}}=\sqrt{1-\text { if }} \int_{\mathrm{o}}^{\mathrm{x}} \mathrm{dx} \cdot \mathrm{k}(\mathrm{x})
\end{aligned}
$$

y la nueva variable $H(x)$ es la amplitud compleja de $h(x)$, siendo $k(x)=\omega / \sqrt{g d(x)}$ el número de onda.

Tomando en cuenta el caso de estudio, una cuenca con anchura constante y que la variaciôn de la profundídad es proporcional a la distancia desde el extremo cerrado de la bah 1a a la boca, tenemos $b=$ constante, $d=x_{0} / L$, donde $x=0$ es la cabeza de la bahỉa, $x=L$ la boca que se comunica al ocêano y $d_{o}$ la profundidad promedio en la boca. La variable $s$, según la ecuación (4), estará dada por:

$$
s=2 k_{0} \sqrt{x L(1-i f)}
$$

siendo $k_{0}=\omega / \sqrt{g_{0}}$

La ecuación diferencial (3) se transforma de acuerdo a las condiciones dadas en el problema, en:

$$
\frac{d^{2} H}{d s^{2}}+\frac{1}{s} \frac{d H}{d s}+H=0
$$

cuya solución es la función de Bessel de orden cero que no diverge en el origen. La solución queda:

$$
\mathrm{H}=\mathrm{C}_{1} \mathrm{~J}_{\mathrm{O}}(\mathrm{s})
$$

donde $\mathrm{C}_{1}$ es una constante $\mathrm{y}_{\mathrm{o}}$ es $1 \mathrm{a}$ funciôn de Bessel de1 primer tipo, de orden cero.

La razón entre las amplitudes en el extremo cerrado $(s=0)$ y en la boca $\left(s=2 k_{o} L \sqrt{1-i f}\right)$ estará dada por:

$$
R=\frac{1}{J_{0}\left(2 k_{o} L \sqrt{1-i f}\right)}
$$

E1 módulo al cuadrado de esta ecuación expresa 1 a función de respuesta de la bahỉa (Fig. 4) y sus mảximos proporcionan los períodos de resonancia.

ANALISIS ESPECTRAL DE LOS DATOS

Para efectuar el análisis espectral se aplica el Método de Máxima Entropía (MEM) elaborado por Burg (1967), obteniéndose el espectro de energía por medio de la estimación de Máxima Entropía, P(f):

$$
P(f)=\frac{P_{N+1} / f_{n}}{\left|1+\sum_{n=1}^{N} \sqrt{n+1} e^{-j 2 \pi f \Delta t}\right|^{2}}
$$


donde

$$
\begin{aligned}
\mathrm{P}_{\mathrm{N}+1} & =\text { error cuadrado medio (o la energia de salida) } \\
\mathrm{f}_{\mathrm{n}} & =1 / 2 \Delta \mathrm{t}=\text { frecuencia de Nyquist } \\
\Delta t & =\text { intervalo de mucstreo } \\
\mathrm{n}+1 & =\text { filtro de predicción de error }
\end{aligned}
$$

Para seleccionar el número de elementos de filtro adecuados se aplica el criterio de Akaike (1969a, b, 1976).

RESULTADOS

Tomando el módulo al cuadrado de la ecuación (8), solución de la ecuación (6), se introducen los valores de los parámetros requeridos para el modelo: $\mathrm{L}=15 \mathrm{~km} \mathrm{y} \mathrm{d}_{\mathrm{o}}=70 \mathrm{~m}$, y se obtienen los periodos de resonancia de la bahí. En la Fig. 2 se muestra la esquematización de la bahía para aplicar e1 modelo, y en la Fig. 4 se muestra la función de respuesta de la bahía en la frecuencia con los valores de $\mathrm{f}=0.18$ y 0.36 para la fricción.

En la tabla No. 1 se dan los períodos de resonancia de la bahía para los diferentes valores de fricción.

Los datos originales fueron filtrados para extraerles las componentes de baja frecuencia y posteriormente se calculó el espectro de energía de la serie de salida del filtro por medio del MEM. E1 espectro resultante se observa en la Fig. 5 y los picos proporcionan los períodos de resonancia de la bahỉ. El espectro de la Fig. 5 (ver notación) presenta dos picos, no obstante, teóricamente aparecen tres picos (Fig. 4, $f=0.18$ ) por lo que la serie original es filtrada con un pasaaltos que convoluciona los pesos del filtro con la serie haciendo que los picos de baja frecuencia se anulen y as 1 , al calcular el espectro de la nueva serie de salida, se detecta el tercer pico (Fig. 5, ver notación). El número de elementos de filtro usados fué de 25.

En 1a Tabla No. 2 se encuentran los resultados obtenidos del anâlisis de la serie y los obtenidos a través del modelo con $\mathrm{f}=0.18$.

\section{DISCUSION}

En la Fig. 4 se nota que con un $f=0.36$ los tres picos se ensanchan, y que el segundo y tercer pico se mueven hacia bajas frecuencias aumentando su período (ver Tabla No. 2). E1 primer pico presenta una ligera disminución en la frecuencia. La amplitud del segundo y el tercer pico disminuye tendiéndo

el tercero a desvanecerse. 


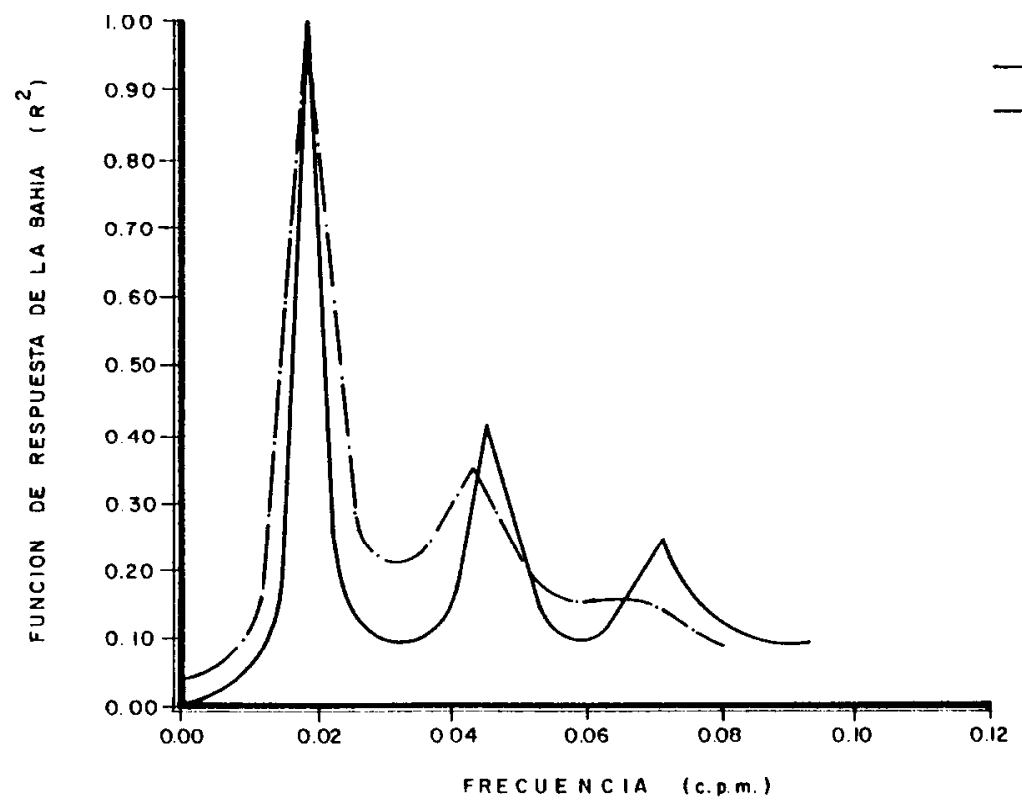

Fig. 4 - FUNCION DE RESPUESTA DE LA BAHIA EN LA FRECUENCIA CON DIFERENTES VALORES DEL PARAMETRO DE FRICCION.

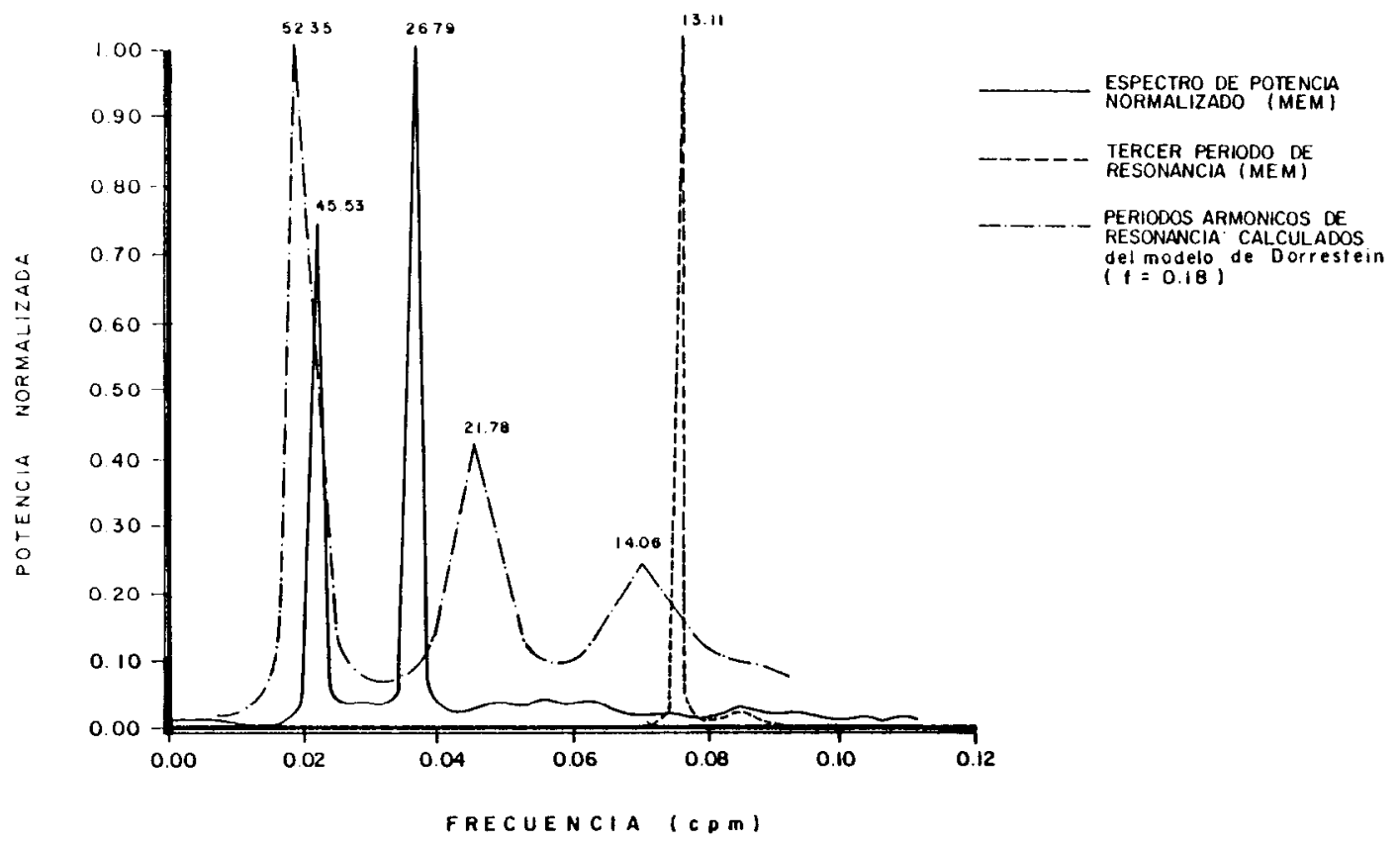

Fig. 5 - COMPARACION ENTRE LOS RESULTADOS OBTENIDOS DEL REGISTRO MAREOGRAFICO $Y$ LOS CALCULADOS TEORICAMENTE 
TABLA No. 1

Períodos de resonancia calculados teóricamente aplicando dos valores del parámetro de fricción.

\begin{tabular}{ccc}
\hline $\begin{array}{c}\text { PERIODOS DE } \\
\text { RESONANCIA }\end{array}$ & $\mathrm{f}=0.18$ & $\mathrm{f}=0.36$ \\
\hline 10. & $52.35 \mathrm{~min}$ & $51.54 \mathrm{~min}$ \\
20. & $21.78 \mathrm{~min}$ & $22.88 \mathrm{~min}$ \\
30. & $14.06 \mathrm{~min}$ & $15.45 \mathrm{~min}$ \\
\hline
\end{tabular}

TABLA no. 2

Períodos de resonancia determinados de los datos observados y a partir del modelo con $\mathrm{f}=0.18$.

\begin{tabular}{ccc}
\hline $\begin{array}{l}\text { PERIODOS DE } \\
\text { RESONANCIA }\end{array}$ & $\begin{array}{c}\text { PERIODOS } \\
\text { OBSERVADOS } \\
\text { (MEM) }\end{array}$ & $\begin{array}{c}\text { PERIODOS } \\
\text { CALCULADOS } \\
\text { (Modelo } \mathrm{f}=0.18)\end{array}$ \\
\hline 10. & $45.53 \mathrm{~min}$ & $52.35 \mathrm{~min}$ \\
20. & $26.79 \mathrm{~min}$ & $21.78 \mathrm{~min}$ \\
30. & $13.11 \mathrm{~min}$ & $14.06 \mathrm{~min}$ \\
\hline
\end{tabular}

En la Fig. 5 se muestra una comparación entre los resultados observados y los calculados del modelo. E1 primer período presenta una desviación a las bajas frecuencias, el segundo período a las altas frecuencias y el tercero también se mueve hacia bajas frecuencias. Por inspección de la Tabla No. 2 se nota que el primero y el tercer período de resonancia teóricos son mayores que los observados debido a su desviación hacia bajas frecuencias.

Si no existiera algún mecanismo disipativo, las amplitudes del nivel dentro de la bahía aumentarían ilimitadamente, por lo que en el modelo se ha incluído un têrmino de fricción.

Dadas las restricciones del modelo que considera una bahia de ancho constante, profundidad promedio en la boca y que las ondas inciden normalmente a la linea de costa puede inferirse que el modelo describe adecuadamente las condiciones resonantes de la bahía (Tabla No. 2). 
La comparación entre 1a energía del espectro resultante y el modelo carece de realidad física, porque el Método de Máxima Entropía posee características no lineales intrínsecas que impiden efectuar una evaluación aceptable de la energia. Este es el principal inconveniente que surge al tratar sertes de datos cortas no estacionarias que requieren alta resolución en la frecuencia.

\section{CONCLUSIONES}

De acuerdo a los resultados que se han obtenido, se concluye que el modelo predice con buena aproximación el comportamiento de la bahỉa en cuanto a sus frecuencias propias de resonancia, aunque no da la información acerca de los factores de amplificación de la amplitud. Al mismo tiempo se ha demostrado que el método de evaluación espectral de Máxima Entropla es muy apropiado, ya que permite estimar las frectiencias resonantes de la bahía aún siendo las características de los datos inapropiadas (serie muy corta y no-estacionaria).

\section{BIBLIOGRAFIA}

AKAIKE, H., 1969a. Fitting autoregressive models for prediction. Ann. Inst. Statistic. Math. 21, 243-247.

-_-_---, 1969b. Power spectrum estimation through autoregressive model fitting. Ann. Inst. Statistic. Math. $21,407-419$.

--_---_, 1970. Statistical predictor identification. Ann. Inst. Statistic. Math. 22, 203-217.

BURG, J.P., 1967. Maximum entropy spectral analysis. Paper presented at the $37 \mathrm{th}$. Annual Int. SEG Meeting, 0klahoma, Oct. 31, 1967. Preprint Texas Instrument, Dallas.

DORRESTEIN, R., 1961. Amplifications of long waves in bays. Eng. Prog. Univ. of Florida, 15(12), December.

RAICHLEN, F., 1970. Tsunamis: Some laboratory and field observations. U.S. Army Coastal Engr. Res. Cont. Corps. Engrs.

SANCHEZ DEVORA, A., 1980. Tsunamis en 1a Costa Occidental de México. Tésis de Maestría, CICESE, $199 \mathrm{pp}$. 